\title{
The Genetic Diversity of the Rice-crayfish Eco-farming Procambarus clarkii in Anhui Province, China
}

\author{
Huan Wang ${ }^{1,2}$, He Jiang ${ }^{1,2}$, Guoqing Duan ${ }^{1,2, *}\left(\mathbb{D}\right.$, Guangtong Song ${ }^{1,2}$, Jun Ling $^{1,2}$, \\ Tingshuang Pan ${ }^{1,2}$, Yuting $\mathrm{Hu}^{1,2}$, Huaxing Zhou ${ }^{1,2}$, Min Yang ${ }^{1,2}$
}

${ }^{1}$ Institute of Fisheries Science, Anhui Academy of Agricultural Sciences, Hefei, Anhui, China, 230031.

${ }^{2}$ Anhui Province Key Laboratory of Aquaculture \& Stock Enhancement, Hefei, Anhui, China, 230031.

\begin{abstract}
How to cite
Wang, H., Jiang, H., Duan, G., Song, G., Ling, J., Pan, T., Hu, Y., Zhou, H., Yang, M. (2022). The Genetic Diversity of the Rice-crayfish Eco-farming Procambarus clarkii in Anhui Province, China. Turkish Journal of Fisheries and Aquatic Sciences, 22(1), TRJFAS19904. http://doi.org/10.4194/TRJFAS19904
\end{abstract}

Article History

Received 28 May 2021

Accepted 10 September 2021

First Online 10 September 2021

\section{Corresponding Author}

Tel.: +8613866682833

E-mail: duangq2010@126.com

\section{Keywords}

Procambarus clarkia

Microsatellite DNA

Genetic diversity

Artificial cultured populations

\begin{abstract}
As a small economic aquatic animal for human consumption, the crayfish Procambarus clarkii breeding was vigorously promoted in Anhui Province. In the present study, genetic analysis was conducted on 12 cultured populations in Anhui Province by microsatellite. The results showed that all twelve populations were showed a high genetic diversity $\left(H_{e}=0.483-0.660, \mathrm{PIC}=0.422-0.588\right)$. Among which the genetic diversity of Quanjiao population is highest, while lowest in Changfeng population. AMOVA analysis showed that most of the genetic variation was found within P. clarkii population $(91.08 \%)$, while only $8.92 \%$ was found among populations. Genetic differentiation and genetic distance analysis showed that the overall differentiation level was moderate $\left(F_{\mathrm{st}}=0.078\right)$. Phylogenetic tree showed that all groups in Taihu and Susong County have a close relationship, Huoqiu and Quanjiao populations formed a sister relationship, and genetic distance between Xuanzhou and other populations was furthest, which mainly related to the parents origin. Additionally, populations experience the bottleneck effect, and inbreeding existed within or among populations, especially in Chngfeng and Xuanzhou populations. The result indicated the germplasm resources of cultured crayfish in Anhui have a tendency of decline, the parents should be updated periodically, and genetic exchanges among the populations should be strengthened.
\end{abstract}

\section{Introduction}

The red swamp crayfish, Procambarus clarkii (Girard, 1852), was originally found in the southeastern United States but introduced to China in the early 20th century, and is nowadays the most cosmopolitan crayfish in the world (Gherardi, 2006; Li et al., 2012). In the 1950s, the pond culture of crayfish had been integrated with other farming operations, such as ricecrayfish co-culture, the mode which can produce both rice and crayfish in the limited space (Mcclain and Romaire, 2004; Si et al., 2017; Sun et al., 2021). As one of the most important freshwater aquaculture resources and well received by consumers in China, crayfish aquaculture has increased rapidly, and crayfish has become the highest-yield freshwater shrimp (Wang et al., 2021).

The artificial breeding of $P$. clarkii began in 1990s in Changfeng County of Anhui province. From 2005 to 2009, the cultivation area and scale gradually expanded, forming three main producing areas: Changfeng County, Quanjiao County and Huoqiu County. Due to its economic and ecological advantages, rice-crayfish ecofarming was greatly promoted. Since 2015, the culture of $P$. clarkii has shown an explosive growth in all cities of Anhui Province, especially in Anqing City. The total 
output of Anhui Province is second in China only to Hubei Province in 2019, which become an important means of rural revitalization.

An important consideration when developing and managing captive populations, however, is the maintenance of genetic diversity to ensure that adequate variation exists to avoid the negative consequences of inbreeding (Miller et al., 2014). The decrease of genetic diversity can lead to the decrease of adaptability and survival of the population (Wang et al., 2017). Studies showed $P$. clarkii genetic diversity has been reduced and the germplasm has been narrowed in China, partly due to a long term of inbreeding, next to the founder effects that resulted from initially small number of individuals, and also for populations of subsequent generations exchange with other populations is minimal (Richards, 2000; Oficialdegui et al., 2019; Sun et al., 2021). Additionally, for captive populations, disorderly introduction, blindly releasing and escape to the natural water also probably occurred. Up to now, the genetic resource data of $P$. clarkii populations in China mainly focus on the wild population (Cao et al., 2010; Li et al., 2012), studies on the cultured populations are still scarce, Zhong et al. (2020) reported genetic diversity of $P$. clarkii populations in different areas of Guangxi Province, and there is no report on the genetic diversity of Anhui cultured populations, it is urgent to clarify the genetic background of the breeding population in Anhui. In the present study, the genetic diversity analysis was conducted on 12 populations of $P$. clarkii in Anhui Province by microsatellite, which aims to enhance the understanding of the genetic difference among various populations and provide basic data for resource protection, breeding and genetic modification, and also for rational and effective artificial breeding and management of the rice-crayfish symbiosis farming.

\section{Materials and Methods}

\section{Sample Collection}

Focusing on Anhui Province, a total of 370 individuals of Procambarus clarkii were collected from 12 artificial cultured populations (Taihu-1, Taihu-2, Taihu-3, Taihu-4, Susong-1, Susong-2, Susong-3, Changfeng, Huoqiu, Chaohu, Xuanzhou and Quanjiao) in different regions of agricultural leading enterprises, breeding with large-scale production farms and foundation seed farms from April to June, 2020 (Figure 1). The muscles tissue samples were quickly taken and cleaned with $0.70 \%$ physiological saline, then stored at $20^{\circ} \mathrm{C}$ in $95 \%$ ethanol.

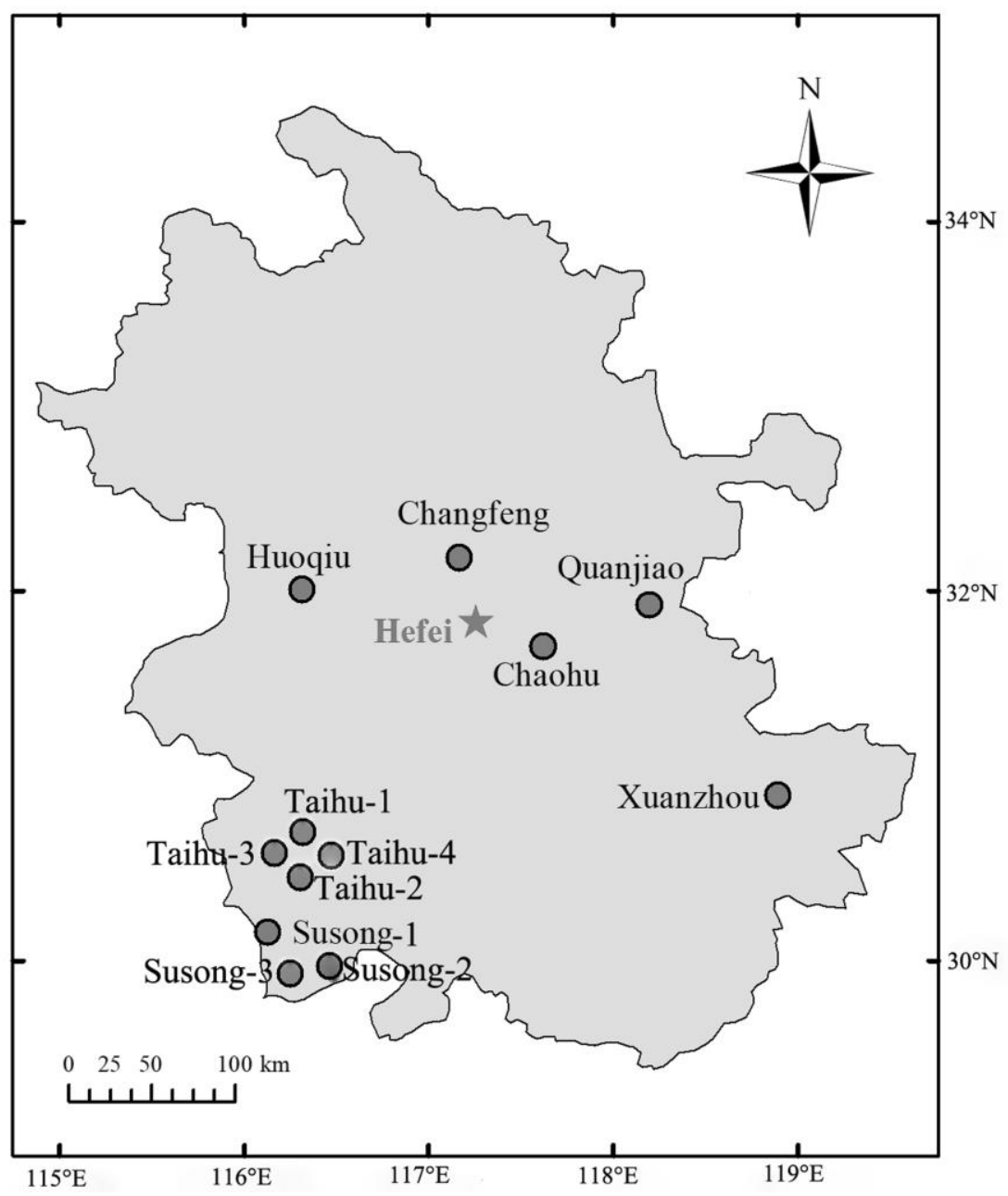

Figure 1. Sampling sites in Anhui Province 


\section{DNA Extraction and Genotyping}

Genomic DNA was extracted using the DNeasy blood and tissue kit (Tiangen, Beijing, China) following manufacturer's instructions. A panel of nine microsatellite markers previously developed for $P$. clarkii were amplified by polymerase chain reaction (PCR) using the annealing temperatures $52-70{ }^{\circ} \mathrm{C}$ (Belfiore and May, 2000). The PCR reactions were conducted using a peltier thermal cycler using a $30 \mu \mathrm{l}$ reaction mixture. Each reaction mixture contained $3 \mu \mathrm{l}$ of $10 \times$ PCR buffer, and final concentrations of $2.5 \mu \mathrm{l}(2.5$ $\mathrm{mmol} / \mathrm{L}) \mathrm{dNTPs}, 1 \mu \mathrm{l}(10 \mu \mathrm{mol} / \mathrm{L})$ each of forward and reverse primer, $0.5 \mu \mathrm{l}(5 \mathrm{U} / \mu \mathrm{l})$ of Taq DNA polymerase (Transgen, Beijing, China), $1 \mu \mathrm{l}(50 \mathrm{ng} / \mu \mathrm{l})$ of template DNA that were added to $30 \mu \mathrm{lddH} 2 \mathrm{O}$. Temperature profiles for the PCR consisted of an initial denaturation at $94{ }^{\circ} \mathrm{C}$ for $5 \mathrm{~min}$, then 32 cycles of $94{ }^{\circ} \mathrm{C}$ for $30 \mathrm{~s}$, annealing at primer specific temperatures for $40 \mathrm{~s}$ at 72 ${ }^{\circ} \mathrm{C}$ for $50 \mathrm{~s}$ and a final extension at $72{ }^{\circ} \mathrm{C}$ for $10 \mathrm{~min}$. Forward primers from each locus were labeled with different fluorescent dyes (Table 1).

The PCR products were separated and sized on an $A B I$ 3730xl automated sequencer with ROX 500 size standard, and the resulting genotype traces scored in GeneMapper 3.7 (all Applied Biosystems). The presence of null alleles, large allele dropouts, scoring of stutter peaks and typographic errors were assessed using a Micro-checker (Van Oosterhout et al., 2004).

\section{Data Analysis}

The microsatellite data were analyzed using webbased Genepop software (http://genepop.curtin.edu.au/), with Markov chain parameters of 10,000 dememorisation, 500 batches and 5000 iterations per batch to determine whether each locus deviated from the Hardy-Weinberg equilibrium and to test the linkage equilibria. The number of alleles $\left(N_{a}\right)$, number of effective alleles $\left(N_{e}\right)$, observed heterozygosity $\left(H_{0}\right)$, expected heterozygosity $\left(H_{e}\right)$, shannon's information index $(I)$, gene flow $\left(N_{m}\right)$ and Nei's genetic distance $\left(D_{a}\right)$ values were calculated using Popgene 1.32, respectively (Nei, 1972; Yeh \& Boyle, 1997).

The genetic differentiation coefficient $\left(F_{s t}\right)$ and genetic variation were analyzed by AMOVA (analysis of molecular variance) using Arlequin 3.5 software (Excoffier \& Lischer, 2010). UPGMA phylogenetic tree based on Nei's genetic distance was constructed by using MEGA 7.0 software (Kumar et al., 2016). The polymorphism information content (PIC) of each locus and each population was calculated using Cervus 3.0 (Kalinowski, 2007).

The genotypes that were determined utilizing COANCESTRY (V1.0.1.1; Wang, 2011) were used to measure relatedness estimates $(R)$ between the populations and within population genotypes using previously described procedures of Wang (2002) and the paternal inbreeding coefficient $(F)$ using previously described procedures of Ritland (1996).

The bottleneck effect was analyzed based on three mutation models, infinite allele model, two phase mutation model and step-wise mutation model using Bottlenecks 1.2. Moreover, mutation-drift equilibrium of the population was analyzed by Sign test and Wilcoxon test to estimate whether the population had heterozygosity excess or deficiency (Luikart \& Cornuet 1998; Piry et al., 1999).

\section{Result}

\section{Summary Statistics}

No evidence of allelic stutter or large allele dropout was found in the dataset, and no null alleles were

Table 1. Summary details of microsatellite loci used in this study.

\begin{tabular}{|c|c|c|c|}
\hline Loci & Primer sequences $\left(5^{\prime} \rightarrow 3^{\prime}\right)$ & $\begin{array}{c}\text { Annealing } \\
\text { temperature }\end{array}$ & Repeat motif \\
\hline \multirow[t]{2}{*}{ PclG02 } & F: CTCCCCATGCACTCTGGCTCTGT_FAM & $66^{\circ} \mathrm{C}$ & $(\text { GATA })_{3}$ GAGAA $(\text { GATA })_{5}$ \\
\hline & R: TGGCGAATTTTGCCTGTTTCTGTC & & \\
\hline \multirow[t]{2}{*}{ PclG03 } & F: CTCTCCACCAGTCATTTCTT-FAM & $52{ }^{\circ} \mathrm{C}$ & $(\mathrm{TCTA})_{20}$ \\
\hline & R: AAGCTTACAATAAATATAGATAGAC & & \\
\hline \multirow[t]{2}{*}{ PclG04 } & F: TATATCAGTCAATCTGTCCAG－FAM & $54{ }^{\circ} \mathrm{C}$ & $\left(\mathrm{TCTA}_{3} \ldots(\mathrm{TCTA})_{2 \ldots}(\mathrm{TCTA})_{29 \ldots}(\mathrm{TCTA})_{2}\right.$ \\
\hline & R: TCAGTAAGTAGATTGATAGAAGG & & \\
\hline \multirow[t]{2}{*}{ PclG07 } & F: CCTCCCACCAGGGTTATCTATTCA-HEX & $63^{\circ} \mathrm{C}$ & $(\mathrm{TCTA})_{8}$ \\
\hline & R: GTGGGTGTGGCGCTCTTGTT & & \\
\hline \multirow[t]{2}{*}{ PclG09 } & F: TATGCACCTTTACCTGAAT-HEX & $60{ }^{\circ} \mathrm{C}$ & $(\mathrm{TCTA})_{14}$ \\
\hline & R: TGTTGGTGTGGTCATCA & & \\
\hline \multirow[t]{2}{*}{ PclG15 } & F: GGCGTGACGCCAACGTGTCTT-HEX & $70{ }^{\circ} \mathrm{C}$ & $(\text { TATC })_{2}$ TGTC $(\text { TATC })_{17}$ TATT $(\text { TATC })_{3}$ \\
\hline & R: GGCTGGCCACTTTGTTAGCCTGAG & & \\
\hline \multirow[t]{2}{*}{ PclG17 } & F: GTCGGGAACCTATTTACAGTGTAT_FAM & $57^{\circ} \mathrm{C}$ & $(\mathrm{TCTA})_{14}$ \\
\hline & R: AAGAGCGAAGAAAGAGATAAAGAT & & \\
\hline \multirow[t]{2}{*}{ PclG29 } & F: GAAAGTCATGGGTGTAGGTGTAAC-HEX & $65^{\circ} \mathrm{C}$ & $(\text { TATC })_{9}$ \\
\hline & R: TTTTTGGGCTATGTGACGAG & & \\
\hline \multirow[t]{2}{*}{ PclG33 } & F: TTCGAGGCGTTGCTGATTGTAAGT_FAM & $68^{\circ} \mathrm{C}$ & $(\mathrm{GT})_{21}$ \\
\hline & R: CAAGGAAGCGTATAGCCGGAGTCT & & \\
\hline
\end{tabular}


detected at any of the nine loci. Almost of all nine loci were found to be highly polymorphic (PIC>0.5). Summary details of the nine microsatellite loci were given in Table 2.

Of the 9 loci assessed for Hardy-Weinberg equilibrium (HWE) were observed using the probability test $(P<0.05)$, five loci in Taihu-4, Huoqiu and Quanjiao, four loci in Changfeng and Chaohu, three in Xuanzhou, Taihu-2, Taihu-3, Susong-1 and Susong-2, two in Susong3 and one locus in Taihu-1 showed significant departures from HWE. When heterozygote excess was assessed using HWE procedures, no disequilibrium was detected in all populations, additionally, no linkage disequilibrium was detected between any loci $(P>0.05)$. The comparison of genetic information of different $P$. clarkii breeding populations in Anhui Province was showed in Table 3. All twelve populations were showed a high genetic diversity $\left(H_{e}=0.483 \sim 0.660, \mathrm{PIC}=0.422 \sim\right.$ 0.588). Among which the genetic diversity of Quanjiao population is highest, while lowest in Changfeng population.

\section{Genetic Variation and Differentiation}

The result of AMOVA showed that the variation among populations is only $8.92 \%$, while variation within populations is $91.08 \%$. Wright (1965) proposed that the genetic differentiation coefficient $F_{\text {st }}<0.05$ was low differentiation, $0.05<F_{s t}<0.15$ was moderate differentiation, $F_{s t}>0.15$ was high differentiation. The whole $F_{s t}$ value is 0.078 , which means a moderate differentiated degree $\left(0.05<F_{s t}<0.15\right)$ (Table 4$)$.

The $F_{s t}$ value of the 12 populations is from 0.0017 to 0.2513 . The lowest genetic differentiation of which was between the populations of Taihu-2 and Susong-2 $\left(F_{s t}=0.002\right)$, with a smallest genetic distance $\left(D_{a}=0.030\right)$, while the highest genetic differentiation of which is between Changfeng and Xuanzhou populations $\left(F_{s t}=0.251\right)$, with a biggest genetic distance $\left(D_{a}=0.481\right)$ (Table 5).

UPGMA phylogenetic tree based on Nei's genetic distance (Nei 1972) indicated the 12 populations have a common root, which can divide the populations into two

Table 2. Genetic information of Procambarus clarkii breeding populations in Anhui Province based on microsatellite markers.

\begin{tabular}{lccccccc}
\hline Locus & $\mathrm{Ho}$ & $\mathrm{He}$ & $\mathrm{PIC}$ & $\mathrm{Na}$ & $\mathrm{Ne}$ & $\mathrm{I}$ & $\mathrm{Nm}$ \\
\hline PcIG02 & 0.5568 & 0.5712 & 0.477 & 6 & 2.3277 & 0.9373 & 2.0035 \\
PcIG03 & 0.6189 & 0.7615 & 0.725 & 14 & 4.1744 & 1.6531 & 2.151 \\
PcIG04 & 0.4784 & 0.6704 & 0.611 & 9 & 3.0255 & 1.2946 & 1.8418 \\
PcIG07 & 0.6000 & 0.6292 & 0.566 & 8 & 2.6905 & 1.2357 & 3.3567 \\
PcIG09 & 0.3297 & 0.7294 & 0.684 & 11 & 3.6814 & 1.5382 & 3.409 \\
PcIG15 & 0.5189 & 0.5623 & 0.511 & 8 & 2.2806 & 1.1106 & 2.9832 \\
PcIG17 & 0.6081 & 0.659 & 0.593 & 9 & 2.925 & 1.2353 & 1.8401 \\
PcIG29 & 0.4703 & 0.7547 & 0.713 & 6 & 4.0605 & 1.4855 & 2.6032 \\
PcIG33 & 0.7703 & 0.6262 & 0.553 & 14 & 2.6691 & 1.2177 & 6.0427 \\
Mean $\pm S D$ & $0.550 \pm 0.123$ & $0.663 \pm 0.074$ & $0.604 \pm 0.083$ & $9.444 \pm 3.005$ & $3.093 \pm 0.713$ & $1.301 \pm 0.223$ & $2.915 \pm 1.249$ \\
\hline
\end{tabular}

Table 3. Comparison of genetic information of Procambarus clarkii breeding populations in Anhui Province.

\begin{tabular}{lcccccc}
\hline Sampling site & $\mathrm{n}$ & $\mathrm{Na}$ & $\mathrm{Ho}$ & $\mathrm{He}$ & $P I C$ & $\mathrm{I}$ \\
\hline Changfeng & 30 & $3.556 \pm 0.882$ & $0.437 \pm 0.208$ & $0.483 \pm 0.193$ & $0.422 \pm 0.158$ & $0.841 \pm 0.318$ \\
Xuanzhou & 30 & $4.333 \pm 1.581$ & $0.507 \pm 0.179$ & $0.570 \pm 0.129$ & $0.498 \pm 0.122$ & $1.018 \pm 0.298$ \\
Chaohu & 30 & $4.667 \pm 1.225$ & $0.507 \pm 0.237$ & $0.627 \pm 0.116$ & $0.560 \pm 0.104$ & $1.145 \pm 0.236$ \\
Huoqiu & 30 & $5.556 \pm 2.297$ & $0.603 \pm 0.198$ & $0.641 \pm 0.118$ & $0.576 \pm 0.118$ & $1.215 \pm 0.321$ \\
Quanjiao & 40 & $5.222 \pm 1.394$ & $0.550 \pm 0.160$ & $0.660 \pm 0.056$ & $0.588 \pm 0.068$ & $1.208 \pm 0.186$ \\
Taihu-1 & 30 & $4.889 \pm 1.453$ & $0.607 \pm 0.185$ & $0.655 \pm 0.069$ & $0.582 \pm 0.074$ & $1.196 \pm 0.187$ \\
Taihu-2 & 30 & $5.222 \pm 1.787$ & $0.622 \pm 0.210$ & $0.628 \pm 0.114$ & $0.556 \pm 0.119$ & $1.156 \pm 0.306$ \\
Taihu-3 & 30 & $4.667 \pm 1.000$ & $0.496 \pm 0.155$ & $0.634 \pm 0.117$ & $0.564 \pm 0.115$ & $1.160 \pm 0.278$ \\
Taihu-4 & 30 & $4.778 \pm 1.481$ & $0.582 \pm 0.185$ & $0.619 \pm 0.108$ & $0.541 \pm 0.117$ & $1.118 \pm 0.296$ \\
Susong-1 & 30 & $5.556 \pm 0.727$ & $0.563 \pm 0.164$ & $0.616 \pm 0.109$ & $0.548 \pm 0.100$ & $1.157 \pm 0.218$ \\
Susong-2 & 30 & $5.000 \pm 1.118$ & $0.541 \pm 0.208$ & $0.618 \pm 0.135$ & $0.549 \pm 0.125$ & $1.139 \pm 0.288$ \\
Susong-3 & 30 & $5.000 \pm 1.323$ & $0.585 \pm 0.149$ & $0.600 \pm 0.097$ & $0.535 \pm 0.100$ & $1.119 \pm 0.247$ \\
\hline
\end{tabular}

Table 4. Analysis of molecular variance (AMOVA) results for Procambarus clarkii in Anhui Province using 9 microsatellite loci.

\begin{tabular}{lcccc}
\hline Source of variation & D.F. & Sum of squares & Variance components & Percentage \\
\hline Among populations & 11 & 193.184 & 0.23539 & 7.84 \\
Within populations & 728 & 2010.433 & 2.76638 & 92.16 \\
Total & 739 & 2203.617 & 3.00177 & 100 \\
\hline
\end{tabular}

Notes : Fst $=0.078$. 
Table 5. Genetic differentiation $F_{s t}$ values and Nei's genetic distance among populations of Procambarus clarkii in Anhui Province.

\begin{tabular}{lccccccccccccc}
\hline & Changfeng & Xuanzhou & Taihu-3 & Taihu-1 & Taihu-4 & Taihu-2 & Susong-3 & Chaohu & Susong-2 & Huoqiu & Susong-1 & Quanjiao \\
\hline Changfeng & $* * * *$ & $\mathbf{0 . 2 5 1}$ & 0.160 & 0.179 & 0.195 & 0.159 & 0.221 & 0.078 & 0.198 & 0.128 & 0.197 & 0.078 \\
Xuanzhou & 0.481 & $* * * *$ & 0.104 & 0.132 & 0.134 & 0.138 & 0.144 & 0.134 & 0.138 & 0.093 & 0.106 & 0.102 \\
Taihu-3 & 0.282 & 0.214 & $* * * *$ & 0.011 & 0.038 & 0.019 & 0.060 & 0.059 & 0.022 & 0.045 & 0.019 & 0.042 \\
Taihu-1 & 0.341 & 0.295 & 0.050 & $* * * *$ & 0.022 & 0.010 & 0.036 & 0.075 & 0.009 & 0.070 & 0.009 & 0.058 \\
Taihu-4 & 0.360 & 0.279 & 0.095 & 0.068 & $* * * *$ & 0.029 & 0.063 & 0.091 & 0.025 & 0.082 & 0.022 & 0.065 \\
Taihu-2 & 0.277 & 0.296 & 0.063 & 0.047 & 0.079 & $* * * *$ & 0.055 & 0.050 & $\mathbf{0 . 0 0 2}$ & 0.070 & 0.023 & 0.047 \\
Susong-3 & 0.420 & 0.292 & 0.133 & 0.090 & 0.137 & 0.122 & $* * * *$ & 0.134 & 0.025 & 0.112 & 0.030 & 0.093 \\
Chaohu & 0.120 & 0.284 & 0.141 & 0.184 & 0.207 & 0.119 & 0.308 & $* * * *$ & 0.084 & 0.041 & 0.094 & 0.029 \\
Susong-2 & 0.369 & 0.287 & 0.067 & 0.044 & 0.070 & 0.030 & 0.066 & 0.191 & $* * * *$ & 0.089 & 0.015 & 0.066 \\
Huoqiu & 0.214 & 0.193 & 0.116 & 0.180 & 0.192 & 0.169 & 0.256 & 0.104 & 0.209 & $* * * *$ & 0.065 & 0.026 \\
Susong-1 & 0.364 & 0.212 & 0.060 & 0.043 & 0.063 & 0.067 & 0.074 & 0.212 & 0.051 & 0.153 & $* * * *$ & 0.064 \\
Quanjiao & 0.120 & 0.218 & 0.109 & 0.153 & 0.156 & 0.119 & 0.213 & 0.081 & 0.158 & 0.077 & 0.153 & $* * * *$ \\
\hline
\end{tabular}

Note: $F_{s t}$ (above diagonal) and Nei's genetic distance (below diagonal).

The bolded values showed highest and lowest $F_{\text {st }}$ values.

clades. The analysis revealed a strong clustering of populations in Taihu and Susong County including Taihu1, Susong-1, Taihu-2, Susong-2, Taihu-3, Taihu-4 and Susong-3, then clustered with the populations of Changfeng, Chaohu, Huoqiu and Quanjiao. Of which, Huoqiu and Quanjiao formed a sister relationship, they were the most related, then clustered with Chaohu and Changfeng. The tree also showed that the genetic distance between Xuanzhou and other groups was furthest (Figure 2).

\section{Partner Relatedness and Inbreeding Coefficient Analysis}

Taihu County and Susong County belong to Anqing City, located in the southwest of Anhui Province. The populations in the two counties revealed a strong clustering according to the above analysis, here, populations from the two counties were combined into Anqing population to calculate the partner relatedness $(R)$ and inbreeding coefficient $(F)$ analysis. The $R$ within each population showed that $R$ value is positive in Changfeng and Xuanzhou, and the value is biggest in Changfeng. However, $R$ value is negative in Chaohu, Huoqiu, Quanjiao and Anqing populations. The result also showed the $F$ value in each of the six populations is positive, with a biggest value in Xuanzhou and smallest value in Anqing (Table 6). The $R$ value between populations is negative except that in Changfeng and Chaohu is positive. The $F$ value in Anqing and other five populations are negative, and also negative in Xuanzhou and Chaohu, Xuanzhou and Quanjiao populations (Table 7).

\section{Mutation-drift Equilibrium Analysis}

According to allele frequency of microsatellite loci and based on three different hypotheses models of IAM, TPM and SMM, mutation-drift equilibrium of $P$. clarkii populations in Anhui Province were tested, and the results are shown in Table 8 . When using Sign test, Xuanzhou and Taihu-4 populations showed significant deviation from mutation-drift equilibrium $(P<0.05)$,
Quanjiao and Taihu-1 populations showed extremely significant deviation from mutation-drift equilibrium under IAM model $(P<0.01)$. Only Susong-1 population was showed significant deviation from mutation-drift equilibrium under TPM model $(P<0.05)$. Only Susong-3 population was showed significant deviation from mutation-drift equilibrium under SMM model $(P<0.05)$.

However, when using two tails for heterozygosity excess and deficiency of Wilcoxon test, Xuanzhou, Taihu-2 and Taihu-4 populations were showed significant deviation from mutation-drift equilibrium $(P<0.05)$, while Chaohu, Quanjiao, Taihu-1 and Taihu-3 showed extremely significant deviation from mutationdrift equilibrium under IAM model $(P<0.01)$. None of any population was deviation from mutation-drift equilibrium under TPM model. However, Susong-1 $(P<0.01)$ and Susong-3 $(P<0.05)$ populations were deviation from mutation-drift equilibrium under SMM model.

\section{Discussion}

\section{Genetic Diversity}

Genetic diversity means genetic variation and adaptation of species' ability to the environment in the long-term evolutionary process (Liu et al., 2020). In general, an invasive alien species often can avoid inbreeding defects and adapt well to a new environment, thereby resulting in high genetic diversity (Zhong et al., 2020). Since multiple paternity was detected in Procambarus clarkii, which is also a compensation mechanism to maintain genetic diversity (Yue et al., 2010; Wang et al., 2017). Studies showed the genetic diversity of 25 wild $P$. clarkii populations in China was still high (Liu et al., 2020). With the promotion of eco-farming $P$. clarkii and rice in China, the production of the cultured crayfish has been greatly increased, while genetic evaluation of the cultured crayfish is less understood.

The genetic diversity of $P$. clarkii cultured populations in different areas of Guangxi Province showed $H_{e}=0.5692-0.6544$, PIC=0.4899-0.5843 (Zhong 


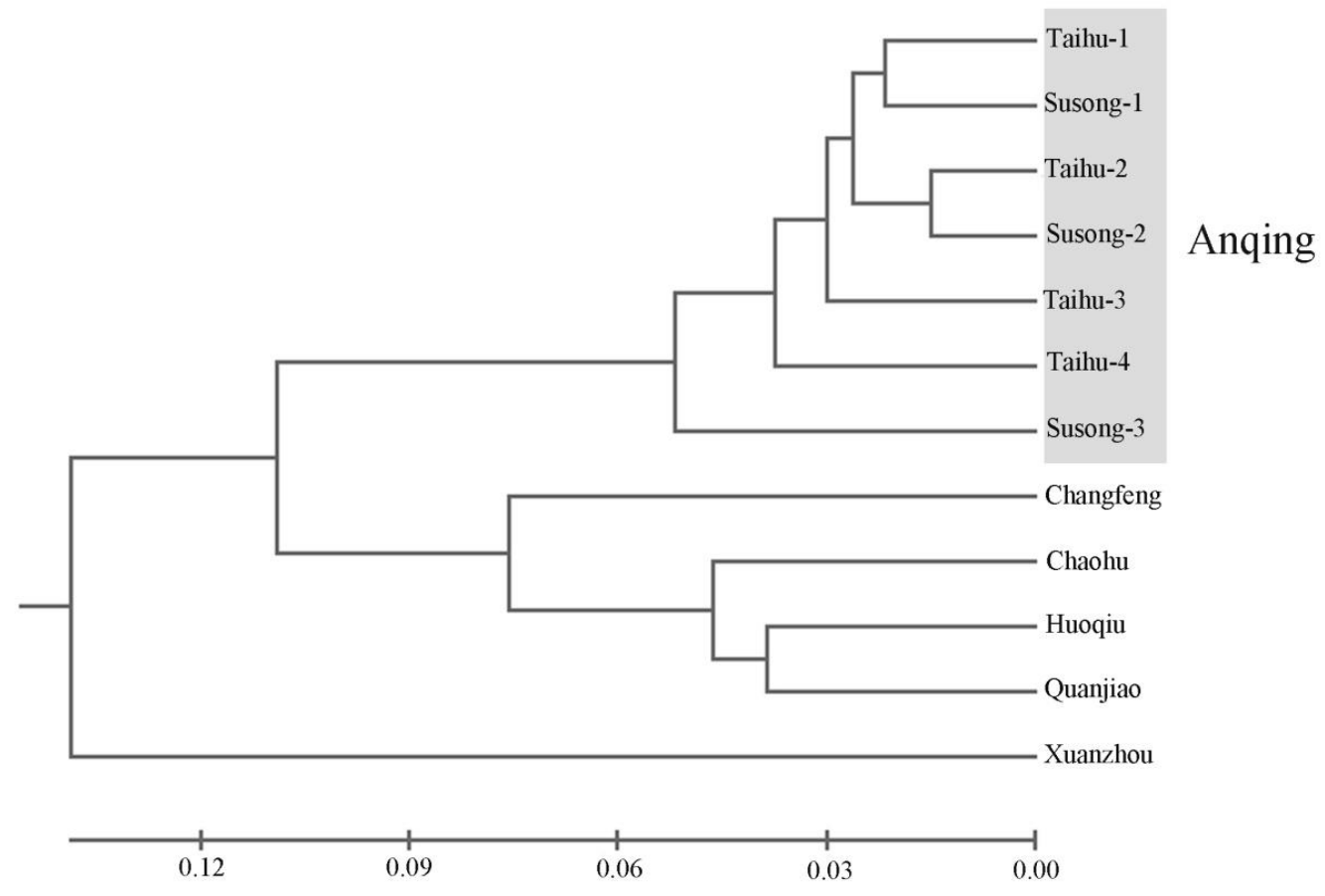

Figure 2. Nei's UPGMA tree of 12 Procambarus clarkii populations based on microsatellites. Note: Bar is the genetic distance.

Table 6. The relationship $(R)$ and inbreeding coefficient $(F)$ within each of the six Procambarus clarkii populations in Anhui Province.

\begin{tabular}{ccccccc}
\hline & Changfeng & Xuanzhou & Chaohu & Huoqiu & Quanjiao & Anqing \\
\hline$R$ & 0.29975 & 0.14865 & -0.01723 & -0.00258 & -0.05079 & -0.00392 \\
$F$ & 0.14764 & 0.18692 & 0.09405 & 0.08605 & 0.04278 & 0.0142 \\
\hline
\end{tabular}

Table 7. The relationship $(R)$ and inbreeding coefficient $(F)$ between the six Procambarus clarkii populations in Anhui Province.

\begin{tabular}{lcccccc}
\hline Populations & Changfeng & Xuanzhou & Chaohu & Huoqiu & Quanjiao & Anqing \\
\hline Changfeng & $* * * *$ & -0.20372 & 0.01984 & -0.05283 & -0.00924 & -0.15993 \\
Xuanzhou & 0.04372 & $* * * *$ & -0.15316 & -0.08773 & -0.13569 & -0.1445 \\
Chaohu & 0.04578 & -0.02187 & $* * * *$ & -0.07932 & -0.07894 & -0.14568 \\
Huoqiu & 0.00058 & 0.00643 & 0.00361 & $* * * *$ & -0.07513 & -0.13527 \\
Quanjiao & 0.03815 & -0.00778 & 0.00677 & 0.00748 & $* * * *$ & -0.13543 \\
Anqing & -0.03127 & -0.02254 & -0.02419 & -0.02297 & -0.02213 & $* * *$ \\
\hline
\end{tabular}

Note: The inbreeding coefficient $(F)$ value is below diagonal and the relationship $(R)$ value is above diagonal.

Table 8 Mutation-drift equilibrium analysis of Procambarus clarkii populations in Anhui Province.

\begin{tabular}{|c|c|c|c|c|c|c|c|c|c|}
\hline \multirow{3}{*}{ Population } & \multicolumn{4}{|c|}{ SIGN TEST } & & & \multicolumn{3}{|c|}{ WILCOXON TEST } \\
\hline & \multicolumn{2}{|c|}{ IAM } & \multicolumn{2}{|c|}{ TPM } & \multicolumn{2}{|c|}{ SMM } & \multirow{2}{*}{$\frac{\text { IAM }}{P}$} & \multirow{2}{*}{$\begin{array}{c}\text { TPM } \\
P\end{array}$} & \multirow{2}{*}{$\frac{\mathrm{SMM}}{P}$} \\
\hline & $H_{E} / H_{D}$ & $P$ & $H_{E} / H_{D}$ & $P$ & $H_{E} / H_{D}$ & $P$ & & & \\
\hline Changfeng & $6 / 3$ & 0.33758 & $5 / 4$ & 0.58229 & $5 / 4$ & 0.58069 & 0.35938 & 0.57031 & 0.73438 \\
\hline Xuanzhou & $8 / 1$ & $0.03754^{*}$ & $7 / 2$ & 0.20160 & $4 / 5$ & 0.27209 & $0.01367^{*}$ & 0.30078 & 0.35938 \\
\hline Chaohu & $8 / 1$ & 0.05029 & $7 / 2$ & 0.20610 & $3 / 6$ & 0.10564 & $0.00586^{* *}$ & 0.12891 & 0.42578 \\
\hline Huoqiu & $7 / 2$ & 0.19690 & $6 / 3$ & 0.44552 & $3 / 6$ & 0.10996 & 0.12891 & 0.65234 & 0.49609 \\
\hline Quanjiao & $9 / 0$ & $0.00615^{* *}$ & $7 / 2$ & 0.20963 & $4 / 5$ & 0.27686 & $0.00195^{* *}$ & 0.12891 & 0.42578 \\
\hline Taihu-1 & $9 / 0$ & $0.00662^{* *}$ & $6 / 3$ & 0.46841 & $4 / 5$ & 0.27730 & $0.00195^{* *}$ & 0.16406 & 0.73438 \\
\hline Taihu-2 & $6 / 3$ & 0.42531 & $5 / 4$ & 0.54626 & $3 / 6$ & 0.10268 & $0.02734^{*}$ & 0.82031 & 0.16406 \\
\hline Taihu-3 & $8 / 1$ & 0.05154 & $6 / 3$ & 0.46564 & $5 / 4$ & 0.53188 & $0.00977^{* *}$ & 0.25000 & 0.91016 \\
\hline Taihu-4 & $8 / 1$ & $0.04457^{*}$ & $5 / 4$ & 0.55201 & $4 / 5$ & 0.27332 & $0.01953^{*}$ & 0.65234 & 0.35938 \\
\hline Susong-1 & $6 / 3$ & 0.46649 & $2 / 7$ & $0.02733^{*}$ & $1 / 8$ & 0.00375 & 0.25000 & 0.25000 & $0.00977^{* *}$ \\
\hline Susong-2 & $7 / 2$ & 0.18918 & $6 / 3$ & 0.46619 & $3 / 6$ & 0.09878 & 0.16406 & 0.49609 & 0.30078 \\
\hline Susong-3 & $6 / 3$ & 0.43315 & $4 / 5$ & 0.29621 & $2 / 7$ & $0.02803^{*}$ & 0.12891 & 0.73438 & $0.01953^{*}$ \\
\hline
\end{tabular}

Note: $H_{E} / H_{D}$ means the ratio of loci number with heterozygosity excess to heterozygosity deficiency; ${ }^{*}$ means significant deviation from mutationdrift equilibrium $(\mathrm{P}<0.05) ;{ }^{* *}$ means extremely significant deviation from mutation-drift equilibrium $(\mathrm{P}<0.01)$. 
et al., 2020). Our present study also showed a similar result, $H_{e}=0.483-0.660, \mathrm{PIC}=0.422-0.588$. However, this result is lower compared with the previous study of wild population. Nine wild populations from the Yangtze, Huaihe, and Xin'anjiang River basins in Anhui Province showed a high genetic diversity $\left(H_{e}=0.78\right.$ ) (Cao et al., 2010). Li et al. (2012) reported 35 wild populations from China showed a relatively high diversity $\left(N_{a}=6.4-11.8\right.$, $H_{e}=0.7002-0.8214$ ), which is lower than that in Louisiana (USA) and Saitama (Japan) population. Some recent studies indicated the genetic diversity of the crayfish in China was decreased, with a medium genetic diversity among 14 populations by using fifty polymorphic SSR markers $\left(H_{e}=0.39, \mathrm{PIC}=0.29\right)$ (Oficialdegui et al., 2019; Sun et al., 2021).

In the present study, the genetic diversity of Changfeng population is lowest, while highest in Quanjiao population. As Changfeng County is first to set up the rice-crayfish eco-farming base in Anhui Province, and become an important base of introduction, like aquaculture enterprises from Chaohu and Huoqiu introduced multiple times. While a self-propagating and self-sustaining model in Changfeng might lead to the decrease of genetic diversity. Quanjiao foundation seed farm is the only one $P$. clarkii foundation seed farm in Anhui Province, which has a mature standard management mode and maintain a high genetic diversity those years.

\section{Genetic Structure, Variation and Inbreeding}

Human mediated dispersal had played an important role in the population expansion and genetic differentiation (Yue et al., 2010). In the present study, parental origin is the primary factor of genetic structure. In recent years, the rice-crayfish eco-farming was vigorously promoted and has become an important source of economy in Taihu and Susong County of Anqing City, situated in southwestern part of Anhui Province. We found populations in Susong and Taihu Counties clustered together, this mainly due to $P$. clarkii in the two counties was introduction from Hubei Province. The whole $F_{s t}$ showed a moderate differentiated degree, which is similar to the study in Guangxi Province (Zhong et al., 2020).

In addition, the population inbreeding coefficient in Anqing city is small compare with other five populations. Genetic distance between Xuanzhou and other populations was furthest, the parental origin of Xuanzhou population is from the local Nanyi Lake, a tributary of the Yangtze River. The partner relatedness of the $P$. clarkii is closest in Changfeng population, and that is also closer in Xuanzhou population. Additionally, there is a risk of inbreeding in each inner-farm especially Xuanzhou population. The long-time sealing culture mode should be change in Changfeng and Xuanzhou populations according to this result.

Commonly, introduced populations carry only a portion of the source population's genetic variability, and genetic variability can be further reduced by stochastic events such as genetic bottlenecks and/or founder effect in new habitats (Almerao et al., 2018). The populations of $P$. clarkii in Anhui Province except Changfeng, Huoqiu and Susong-2 populations probably suffering bottleneck effect under IAM, TPM and SMM by Sign test and Wilcoxon test. However, studies have shown that many microsatellite data are more in line with TPM model, which has been recommended to test the population bottleneck effect (Cornuet et al., 1996). In the present study, mutation-drift had little impact on the populations if only use TPM model.

\section{Conclusion}

In conclusion, the present study is investigated the genetic diversity and structure of artificial cultured populations located in different areas of Anhui. The results showed the genetic diversity of the cultured $P$. clarkii is lower than wild populations in China, and also lower than that in USA and Japan. Particularly, there is a need to improve the germplasm resources of $P$. clarkii in Changfeng and Xuanzhou. Genetic variation is mainly within populations, and genetic differentiation is moderate. Meanwhile, inbreeding existed within or among populations. The result suggested that the cultured P. clarkii populations in Anhui Province should update the parents and strengthen genetic exchanges among the populations, for example, introduce good varieties from other provinces or abroad to decrease the inbreeding coefficient and maintain the genetic diversity of the populations.

\section{Ethical Statement}

The guidelines established by the Administration of Affairs Concerning Animal Experimentation state that approval from the Science and Technology Bureau of China and the Department of Wildlife Administration is not necessary when the animals in question are neither rare nor near extinction (first- or second-class state protection level). Therefore, approval was not required for the artificial cultured Procambarus clarkia conducted in this study.

\section{Funding Information}

This study was funded by the Competitiveness Improvement Project of Modern Agricultural Industrial Technology System in Anhui Province: Technological Action Project of Enhancing Competitiveness of rice and fishery comprehensive planting and breeding industry of Anhui province in 2019, Anhui Province Science and Technology Major Project: Integrated Demonstration and Industrial Poverty Alleviation of Key Technologies of rice and Shrimp Ecological Planting and breeding (201903B06020015), and Key Research and Development Plan of Anhui Province (201904a06020032). 


\section{Author Contribution}

All authors are responsible for the general design of the manuscript. WH analyzed the data and wrote the manuscript. JH contributed to data analysis and revised the manuscript. SG, LJ and PT collected the samples and revised the manuscript. $\mathrm{HY}, \mathrm{ZH}$ and $\mathrm{YM}$ revised the manuscript. DG supervised the whole project. All authors contributed on specific aspects.

\section{Conflict of Interest}

The authors declare no conflict of interest.

\section{References}

Almerao, M.P., Delaunay, C., Coignet, A., Peiro, D.F., Pinet, F., \& Grosset, C.S. (2018). Genetic diversity of the invasive crayfish Procambarus clarkii in France. Limnologica. 69, 135-141. https://doi.org/10.1016/j.limno.2018.01.002.

Belfiore, N.M., \& May, B (2000). Variable microsatellite loci in red swamp crayfish, Procambarus clarkii, and their characterization in other crayfish taxa. Mol. Ecol. 12, 2231-2234. https: //doi.org/10.1046/j.1365-294x.2000.105339.x.

Cao, L., Zhou, L., \& Zhang, B. (2010). Genetic patterns of an invasive Procambarus clarkii population in the three river basins of Anhui Province. Biodivers. Sci. 18, 398407.http://www.biodiversityscience.net/EN/Y2010/V18 $/ 14 / 398$

Cornuet, J.M., \& Luikart, G. (1996). Description and power analysis of two tests for detecting recent population bottlenecks from allele frequency data. Genet. 144, 2001-2014.

https://www.genetics.org/content/genetics/144/4/200 1.full.pdf.

Excoffier, L., \& Lischer, H.E.L. (2010). Arlequin suite ver 3.5: a new series of programs to perform population genetics analyses under Linux and Windows. Mol. Ecol. Resour. 10, 564-567. https://doi.org/10.1111/j.1755-0998.2010.02847.x

Kalinowski, S.T. (2007). Revising how the computer program CERVUS accommodates genotyping error increases success in paternity assignment. Mol. Ecol. 16, 10991106.https://doi.org/10.1111/j.1365294x.2007.03089.x

Kumar, S., Stecher, G., \& Tamura, K. (2016). MEGA7: molecular evolutionary genetics analysis version 7.0 for bigger datasets. Mol. Biol. Evol. 33, 1870-1874. https://pubmed.ncbi.nlm.nih.gov/27004904/

Li, Y., Guo, X., Cao, X., Deng, W., Luo, W., \& Wang, W. (2012). Population genetic structure and post-establishment dispersal patterns of the red swamp crayfish Procambarus Clarkii in China. Plos. One. 7, e40652. https://journals.plos.org/plosone/article?id=10.1371/jo urnal.pone.0040652

Liu, F., Qu, Y.K., Geng, C., Wang, A., Zhang, J., Li, J., Chen, K., Liu, B., Tian, H., Yang, P., \& Yu, Y. (2020). Analysis of the population structure and genetic diversity of the red swamp crayfish (Procambarus clarkii) in China using SSR markers. Electron. J. Biotechn. 47, 59-71. https://www.sciencedirect.com/science/article/pii/S07 17345820300312

Luikart, G., \& Cornuet, J. (1998). Empirical evaluation of a test for identifying recently bottlenecked populations from allele frequency data. Conserv. Biol. 12, 228-237. https://www.jstor.org/stable/2387479

Miller, M.P., Pratt, G.F., Mullins, T.D., \& Haig, S.M. (2014). Comparisons of genetic diversity in captive versus wild populations of the federally endangered Quino checkerspot butterfly (Euphydryas editha quino Behr; Lepidoptera: Nymphalidae). P. Entomol. Soc. Wash. 116, 80-90.

https://www.mendeley.com/catalogue/b9052bb7904e-3401-a144-f7b193edb358/

Nei, M. (1972). Genetic distance between populations. Am. Nat. 106, 283-292.

https://www.researchgate.net/publication/200101860 Genetic_Distance_between_Populations

Oficialdegui, F.J., Clavero, M., Sánchez M.I., Green, A.J., Boyero, L., Michot, T.C., Klose K., Kawai T., \& Lejeusneet C. (2019). Unravelling the global invasion routes of a worldwide invader, the red swamp crayfish (procambarus clarkii). Freshwater Biology, 64, 13821400. https://doi.org/10.1111/fwb.13312.

Piry, S., Luikart, G., \& Cornuet, J.M. (1999). Computer note. Bottleneck: a computer program for detecting recent reductions in the effective size using allele frequency data. J. Hered. 90, 502-503. https://academic.oup.com/jhered/article90/4/502/860 820

Richards, C.M. (2000). Inbreeding depression and genetic rescue in a plant metapopulation. Am. Nat. 155, 383394. https://doi.org/10.1086/303324

Ritland, K. (1996). Estimators for pairwise relatedness and individual inbreeding coefficients. Genet. Res. 67, 175185. https://doi.org/10.1017/S0016672300033620

Si, G., Peng, C., Yuan, J., Xu, X., Zhao, S., Xu, D., \& Wu, J. (2017). Changes in soil microbial community composition and organic carbon fractions in an integrated rice-crayfish farming system in subtropical China. Sci. Rep. 7, 2856. https://doi.org/10.1038/s41598-017-02984-7

Sun, J., Peng, G., Xiong, L., Tan, C., Li, Y., \& Bai, X. (2021). Genome-wide SSR marker development and application in genetic diversity analysis of the red swamp crayfish, Procambarus clarkii (Girard, 1852) in China. Crustaceana, 94, 189-205. https://doi.org/10.1163/15685403-bja10076

Van Oosterhout, C., Hutchinson, W.F., Wills, D.P., \& Shipley, P. (2004). MICRO-CHECKER: software for identifying and correcting genotyping errors in microsatellite data. Mol. Ecol. Notes 4, 535-538. https://doi.org/10.1111/j.1471-8286.2004.00684.x

Wang, H., Yan, P., Zhang, S., Sun, L., Ren, M., Xue, H., Zhang, F., Wu, R., \& Wu, X. (2017). Multiple paternity: A compensation mechanism of the Chinese alligator for inbreeding. Anim. Reprod. Sci. 187, 124-132.

https://www.sciencedirect.com/science/article/abs/pii/ S0378432017306292

Wang, J.L. (2011). Coancestry: a program for simulating, estimating and analysing relatedness and inbreeding coefficients. Mol. Ecol. Resour. 11, 141-145. https://doi.org/10.1111/j.1755-0998.2010.02885.x

Wang, J. (2002). An estimator for pairwise relatedness using molecular markers. Genet. 160, 1203-1215. https://doi.org/10.1089/109065702760093951

Wright, S. (1965). The interpretation of population structure by F-statistics with special regard to systems of mating. Evolution. 3, 395-420. https://doi.org/10.1111/j.1558-5646.1965.tb01731.x 
Yeh, F.C., \& Boyle, J. (1997). POPGENE, the user-friendly shareware for population genetic analysis. Mol. Biol. Biotechnol. 434, 724-731.

Yue, G., Li, J., Wang, C., Xia, J., Wang, G., \& Feng, J. (2010). High prevalence of multiple paternity in the invasive crayfish species, Procambarus clarki. Int. J. Biol. Sci. 6, 107-115. http://pdfs.semanticscholar.org/317e/92a8379ea4afb8 222c9c5e61f6ca724fca69.pdf

Yue, G., Li, J., Bai, Z., Wang, C., \& Feng, F. (2010). Genetic diversity and population structure of the invasive alien red swamp crayfish. Biol. Invasions. 12, 2697-2706. https://link.springer.com/article/10.1007/s10530-0099675-1

Zhong, Y., Tang, Z., Huang, L., Wang, D., \& Lu, Z. (2020). Genetic diversity of Procambarus clarkii populations based on mitochondrial DNA and microsatellite markers in different areas of Guangxi, China. Mitochondrial. DNA. A. $31,48-56$

https://www.tandfonline.com/doi/full/10.1080 /24701394.2020.1721484 